\title{
Usage of healthcare services and preference for mental healthcare among older Somali immigrants in Finland
}

\section{Mölsä, Mulki Elmi}

2019-08-18

Mölsä , M E , Tiilikainen , M \& Punamäki , R-L 2019 , ' Usage of healthcare services and preference for mental healthcare among older Somali immigrants in Finland ', Ethnicity \& Health , vol. 24 , no. 6 , pp. 607-622 . https://doi.org/10.1080/13557858.2017.1346182

http://hdl.handle.net/10138/312037

https://doi.org/10.1080/13557858.2017.1346182

unspecified

acceptedVersion

Downloaded from Helda, University of Helsinki institutional repository.

This is an electronic reprint of the original article.

This reprint may differ from the original in pagination and typographic detail.

Please cite the original version. 
Usage of healthcare services and preference for mental healthcare among older Somali immigrants in Finland

Mulki Mölsä

University of Helsinki, Department of Public Health, Finland

mulkielmi60@gmail.com

Marja Tiilikainen

University of Helsinki, Department of Social Research, Finland

marja.tiilikainen@helsinki.fi

Raija-Leena Punamäki

University of Tampere, Faculty of Social Sciences, Psychology, Finland, and University of Sevilla, Department of Evolution and Education Psychology, Spain

raija-leena.punamaki@staff.uta.fi

Correspondence: Marja Tiilikainen, Department of Social Research, Unioninkatu 35, P.O.Box 18, 00014 University of Helsinki, Finland, e-mail marja.tiilikainen@helsinki.fi. 


\begin{abstract}
Objectives: The aims of the study were, first, to describe and analyze healthcare services utilization patterns of older immigrants in Finland, and particularly to compare the availability and accessibility of health services between older Somalis and Finns. The second aim was to examine the preferences for mental healthcare within the group of Somalis. The third aim was to test the existence of a service usage gap expected to be characteristic of the Somali group, in which high levels of mental health problems occur alongside simultaneous low levels of mental health service usage.
\end{abstract}

Design: The participants were 256 men and women between the ages of 50-85; half were Somali migrants and the other half Finnish matched pairs. The participants were surveyed regarding their usage of somatic, mental, and preventive health services, as well as symptoms of depression, general distress, and somatization. The Somali participants were also surveyed regarding their usage of traditional healing methods and preferences for mental healthcare.

Results: The Somali group had significantly lower access to personal/family doctors at healthcare centers as well as a lower availability of private doctors and occupational health services than the Finns. Instead, they used more nursing services than Finnish patients. The Somali participants attended fewer age-salient preventive check-ups than the Finns. The majority of the Somalis preferred traditional care, most commonly religious healing, for mental health problems. The hypothesized service gap was not substantiated, as a high level of depressive symptoms was not associated with a low usage of health services among the Somalis, but it was found unexpectedly among the Finns.

Conclusion: Our findings call for culturally appropriate general and mental health services for older immigrants, which requires awareness of clients' preferences, needs, and alternative healing practices. Somali participants encountered institutional barriers in accessing healthcare, and they preferred informal mental healthcare, especially religious healing instead of Western practices.

Key words: healthcare services usage, immigrants, older Somalis, mental health, traditional and religious healing, Finland 


\section{Usage of healthcare services and preference for mental healthcare among older Somali immigrants in Finland}

\section{Introduction}

Research suggests that migrants and refugees in Western countries use fewer healthcare services, particularly mental health and preventive services, than the native population (Diaz and Kumar 2014; Morrison et al. 2012; Uiters et al. 2009; Wittig et al. 2008). The lower rate of usage of mental health services is also prevalent in countries where there is a cost-free universal healthcare system such as in Canada and Finland (Fenta, Hyman, and Noh 2007; Gissler et al. 2006; Kirmayer et al. 2011; Koponen et al. 2015, 177-179). The low usage of available services is considered paradoxical, as immigrants, and especially refugees, show a high prevalence of mental health problems, including depression, anxiety, and somatization disorders (Close et al. 2016; Diaz and Kumar 2014; Fazel, Wheeler, and Danesh 2005). This is referred to as the service usage gap among refugees and migrant populations.

Commonly-documented reasons for migrants' lower usage rates of mental health services are cultural and linguistic barriers (Kirmayer et al. 2011; Koopmans et al. 2013), which create great challenges to healthcare providers in receiving countries. One way to meet these challenges is to increase healthcare personnel's cultural literacy by improving their knowledge and understanding of immigrant populations' health needs, values, and preferences (Sandvik, Hunskaar, and Diaz 2012). Thus, research about the specific needs and service preferences of various migration groups is needed, as well as research regarding the connections between their health status and use of healthcare services (Fenta, Hyman, \& Noh 2007; Wittig et al. 2008). Accordingly, this study analyses service utilization patterns, and, in particular, compares the availability and accessibility of 
health services for older Somali and Finnish participants. In addition, it explores the preferences for mental health treatment among older Somali immigrants in Finland.

\section{Immigrant health and service usage}

Although research shows increased risks of psychiatric disorders and mental health problems among migrants and refugees upon settling in Western countries, the severity varies greatly according to demographic and cultural factors (Close et al. 2016; Ladin and Reinhold 2013; Vervliet et al. 2014). Notably, a systematic review revealed that depression varied between $8-12 \%$ in native populations, while the variation was between $4-40 \%$ in immigrants and refugees (Close et al. 2016). Women, elderly, and migrants with low education levels are considered to be the most vulnerable to mental health problems, especially depression (Mirsky et al. 2011). Concerning the role of receiving society, immigrants show better mental health in countries with a high gross national product (GNP), while GNP does not impact refugees' mental health (Close et al. 2016). A Finnish epidemiological study (MAAMU) on immigrants showed the relevance of the country of origin; Kurdish immigrants, especially women, showed significantly worse mental health than the Finnish population, whereas the Somali population's mental health did not differ from the native population's mental health status (Castaneda et al. 2012).

Research confirms that migrants in Finland use fewer mental health and preventive health services than the native population, repeating a pattern found in other receiving countries (Castaneda et al. 2012; Gissler et al. 2006; Koponen et al. 2015). The MAAMU study further showed that, although migrants, especially Somalis, have confidence in the Finnish health service system, mental health and preventive services reach only a small proportion of those needing these services (Castaneda et al. 2012). A study in the United States also found that, in spite of the availability of preventive services, Somali migrants used fewer preventive care services than did the natives (Morrison et al. 2012). In the United Kingdom, Somalis scarcely used mental health 
services, in spite of their relatively high levels of psychological distress (McCrone et al. 2005; Warfa et al. 2006). In addition, there were migrants who fell completely outside of the existing health and social services, particularly asylum seekers and undocumented migrants, despite their especially high needs (Gerritsen et al. 2006).

Yet, research shows great differences in healthcare use among immigrants related to the type of services used and the countries inhabited. A Norwegian register study found that older migrants between the ages of 60-65 used fewer general practitioner (GP) services than the same-age native population, and migrants between the ages of 65-80 used more (Diaz and Kumar 2014). Gender also intersects with ethnic background and impacts the use of mental health services. In Finland, for example, migrant women have been found to use mental health services more frequently compared to migrant men, echoing rates of usage among the general Finnish population. However, among certain African groups, such as Somalis, the use of mental health services is significantly lower than among the Finnish women in general (Koponen et al. 2015, 177-178). Immigrants in many European countries have been found to use services provided by general practitioners and emergency healthcare doctors (Sandvik, Hunskaar, and Diaz 2012). Yet, generally, immigrants seldom use psychiatric or therapy services (Castaneda et al. 2012; Fenta, Hyman, and Noh 2007).

Some research is available on migrants' use of alternative treatments and the services of spiritual/religious healers as substitutes for the lack of culturally-accepted mental healthcare providers in Western countries (Johnsdotter et al. 2011; Wedel 2011; for a transnational context, see Tiilikainen and Koehn 2011). In addition, mental disorders are not always recognized, and treatment is not always sufficient, as indicated by evidence from Finland and Canada (Castaneda et al. 2012; Fenta, Hyman, and Noh 2007).

The low or unsuitable use of mental health services despite severe health problems is conceptualized as a service usage gap, which has been attributed to distinct cultural health beliefs 
and the dissimilar values and customs of immigrants and refugees, on the one hand, and to characteristics of the healthcare system, on the other (Johnsdotter et al. 2011; Wittig et al. 2008). Research confirms that Somali immigrants consider a holistic approach, including harmonious social relations as well as sensitivity to cultural and religious dimensions of health and healing, as a basis for good mental health (Johnsdotter et al. 2011; Mölsä, Hjelde, and Tiilikainen 2010; Wedel 2011). Subsequently, effective healing should focus on benevolent changes in social and spiritual relations (Guerin et al. 2004; Kuittinen et al. 2014).

Healthcare providers may be culturally illiterate or insensitive and unconsciously reflect the discrepancies between the Western biomedical healing model's understanding of mental health problems and immigrants' social, religious, and traditional explanations (Tiilikainen and Koehn 2011; Whitley, Kirmayer, and Groleau 2006). A study reported that Somalis living in Finland felt that healthcare professionals often lacked an understanding of their life situations and needs (Somalis in Helsinki 2013, 88-92). Somali respondents regarded communication with medical professionals as especially problematic, partly due to the different cultural ways of expressing and understanding illnesses.

\section{The context of the present study}

The population with immigrant backgrounds in Finland is much smaller than the populations in the other Nordic countries of Sweden, Norway, and Denmark. Somalis constitute the third-largest immigrant group in Finland, following migrants originating from Russia or the former Soviet Union and Estonia. The number of Somali-language speakers in 2015 was 17,871, with almost 48\% female, and there were only 1,273 people who were 50 years old or older (Statistics Finland 2016). The Somali immigrants in Finland are the largest African-origin group, the largest ethnic group with a refugee background, and also the largest Muslim group. Many Somali immigrants have reported experiences of racial discrimination and adjustment problems (Castaneda et al. 2012; Somalis in 
Helsinki 2013). In addition, the unemployment rate has remained high; in 2012, approximately $51 \%$ of Somali immigrants were unemployed, and only $20 \%$ of the Somalis belonging to the working-age population (15-64 years old) were employed (Joronen and Mohamed 2015, 17-18).

Healthcare services differ greatly in Finnish and Somali contexts and, thus, the pathways to care and the healthcare decision-making processes differ. Even before the Somali civil war in the late 1980s, the healthcare system was not functioning properly in Somalia due to a lack of resources. Psychiatric and preventive healthcare services were almost non-existent, even in urban areas. There were mental hospital wards in only three cities: Berbera, Hargeisa, and Mogadishu (Omar 1986). The use of traditional healing methods, such as religious healing practices and spirit possession rituals, particularly as treatments for mental distress, has been very common (Cavallera et al. 2016; Johnsdotter et al. 2011; Mölsä, Hjelde, and Tiilikainen 2010). For example, saar (zar) refers to a spirit possession ritual known in Somalia and some other countries in the Horn of Africa (Lewis, Al-Safi, and Hurreiz 1991). Characteristically, Somalis use healthcare in a pluralistic way. Many actors are involved in helping those seeking care and taking part in decision-making. Wider social networks interpret health-related information and influence attitudes towards, as well as acceptance and use of, services. A Somali proverb, "Nin bukaa, boqol u talise" - A sick man is advised by a hundred - illustrates well this communal help-seeking behavior (Serkkola 1994, 56$58)$.

In Finland, the healthcare system is mainly publicly funded and provides universal healthcare provided by the National Health Insurance system (NHI) to everyone residing in Finland, regardless of nationality. The healthcare services are divided into a municipal healthcare system, private health care services, and occupational health services provided by employers. In the public municipal healthcare centers that are almost free and open to everyone, a client will be first referred to either a nurse or a general practitioner. The GP will further decide if special care is needed. At a healthcare 
center, a personal/family doctor is responsible for families in a certain geographical area (Wahlbeck et al. 2008). Migrants in Finland mostly use public healthcare, as many of them are unemployed and therefore cannot afford to use private healthcare or utilize occupational healthcare services (Castaneda et al. 2012).

\section{Research aims}

The first aim of this study was to analyze whether elderly Somali immigrants and Finnish natives differ in their healthcare service utilization. The analysis focused on the accessibility and use of general and specialized healthcare, mental health services, occupational health, and preventive care. Secondly, within the Somali group, we analyzed the preferences for mental healthcare and healing practices for individuals suffering psychiatric distress. The third aim was to test whether there is a service usage gap among elderly Somali immigrants, indicated by a high level of mental health problems (depressiveness, psychological distress, and somatization) accompanied by a low level of service usage.

\section{Data and methods}

\section{Participants and procedures}

The study participants were 128 Somalis and their 128 matched Finnish pairs, all between the ages of 50-80 and all living in Finland. The groups were matched according to their gender, age, education, and marital status (reference was omitted for anonymity). In both groups, there were 75 female and 53 male participants. The basic sample were Somalis living in the metropolitan area of Helsinki (N=307, according to the National Register), and, of this basic sample, every second participant was randomly selected to be interviewed. Fifteen individuals of the eligible sample $(\mathrm{N}=154)$ were excluded due to hospitalization or absence, two were dead, and nine refused to participate. No other exclusion criterion was used. The National Register provided contact information, and the participants were contacted by phone and information letters, as well as 
through social networks and unofficial inquiries. The majority of Somali women chose to be interviewed in their homes, while men often preferred other interview places, such as cultural centers or coffee shops. The Somali data were collected in 2007, and the Finnish data were taken from the National Health Survey collected in 2000-2001 (Heistaro 2008). Each Somali participant was matched with a Finnish counterpart according to gender, age (dichotomy variable: 1. 50-59 years; 2 older), and education (dichotomy variable 1 . Polytechnic or university; $0=$ lower level).

The first author organized and supervised the data collection. Eight Somali-speaking interviewers (five men and three women) conducted the data collection. They were trained by two experts (a medical doctor and a psychologist) regarding interview techniques, appropriate ways to approach families, and content of questions. All eight interviewers were known and well-respected members of the Somali community in Finland and had educational backgrounds in social, pedagogic, and nursing sciences. During the fieldwork, the first author held regular supervision meetings where the interviewers could discuss their views and experiences, as well as any problems they encountered.

The questionnaire was first translated from Finnish to Somali by a professional translator. The translation was then double-checked by two Somali language experts - linguists who participated in the creation of written Somali language in 1974. No back translation was conducted. The batteries of questions regarding availability and accessibility of healthcare services and mental health chosen for this study are widely used in international surveys as well as in Finnish national surveys. Additional questions were specifically developed for the Somali group concerning their preferences for mental healthcare and traditional healing methods. To ensure the cultural appropriateness of the questionnaire, a pilot testing of the interview protocol was conducted among ten Somali individuals who were not part of the study. 
The Epidemiological Ethics Committee of the Hospital District of Helsinki and Uusimaa, Finland has approved the trial study protocol concerning mental health (3/9/3100500), and the National Institute for Health and Welfare (NIHW) approved the specific part of the questionnaire for refugees (March $\left.5^{\text {th }}, 2007\right)$. All participants were informed about the aims and procedure of the study, and an informed consent was obtained before active participation commenced.

\section{Measures}

Somatic health service availability and accessibility was measured by asking the following questions. "Do you have, at your municipality health center, a so-called personal doctor (family doctor)?" Allowable answers were: yes, no, and cannot tell. "Do you seek help from this doctor when you need medical advice?" This question allowed for either a yes or no response. "Do you have some other doctor from whom you primarily seek help and advice? Who is this doctor?" There were six allowable responses to this question: health center doctor (GP); occupational healthcare doctor; private doctor; doctor in hospital outpatient department; doctor who is an acquaintance or relative; some other doctor. "Do you have a certain nurse whom you contact when you need help or advice concerning your health?” Answers could be either yes or no.

Mental health service usage during last year was determined by asking whether participants had, during the past 12 months, used any health services because of mental problems, with either yes or no as the response. Further, a specified response alternative was assessed to determine where participants had sought care, with the following responses allowed: a health center, an occupational health service, a mental health center or psychiatric outpatient department, an A-clinic, a family advice bureau or a child guidance center, a private consultation of a doctor or a psychologist, a psychiatric hospital, another hospital, a rehabilitation center, or somewhere else (with a follow-up question requesting the location). Participants were also asked how many times in the past 12 months they had used those healthcare services. Those who confirmed their treatment were asked 
whether their treatment was completed, whether it comprised psychotherapy, and who had treated them, with possible responses of psychiatrist, other doctor, nurse or a public health nurse, other professional, and traditional healer. Participants were also asked whether their treatment included pharmacotherapy and whether the treatment had helped them, as well as the extent to which the treatment had helped.

To investigate the use of preventive health services, the participants were asked about several gender-based treatments. Women were asked about mammography and palpations of the breasts, Pap test, and gynecological examination. Men were asked about the ultrasound of the prostate and the PSA, which is a value measured in the blood sample to check prostate function. In Finland, mammography, Pap tests, and PSA tests are routine free health screenings for people over 50 years old.

Preferences for healthcare were solicited only from the Somali elderly. The questions concerned alternative healthcare services used in Finland or abroad. In addition, participants were asked whether they could recall and describe whether somebody dear to them was suffering from mental illness or mental health problems, as well as their preferable first choice for a place of care in such an circumstance. Allowed responses were religious expert/faith healer, traditional (saar) healer, herbalist, Finnish healthcare, elsewhere - specify.

Mental health was measured using a variety of instruments. Depression symptoms were documented using the 21-item Beck Depression Inventory (BDI, Beck et al. 1961), involving culturally-relevant dimensions of somatic-affective and psychological-cognitive dimensions (Kuittinen et al. 2014). Psychiatric distress was calculated using the General Health Questionnaire, (GHQ-12, Goldenberg et al. 1997), and somatization was evaluated with the 13-item Symptom Checklist-90-Revised, (SCL-90-R, Derogatis 1992), which depicts symptoms such as faintness and dizziness, soreness of muscles, and unspecified pains and aches. 


\section{Results}

\section{Descriptive results}

Table I presents the background variables in the Somali and Finnish groups. Results confirm that the matching of the groups was successful, because the Somali and Finnish groups did not differ in the gender, age, civic status, or education. The sample included slightly more women (59\%), and the majority (61\%) were between 50-59 years of age. Similarly, $62 \%$ were married, and the others were widowed, divorced, or never married. The groups differed significantly in employment, as only $28.2 \%$ of the Somalis and $78.1 \%$ of the Finns were fully employed.

Approximately half of the Somali participants (47.7\%) had a permanent residence permit in Finland, and $19.7 \%$ had Finnish nationality, while the rest had a residence permit by humanitarian law $(16.1 \%)$, family member status $(7.3 \%)$, or other type of permit $(9.2 \%)$. The participants' length of stay in Finland varied between 1-20 years. All Somali participants were born in Somalia; of those, $40.1 \%$ had lived in Finland between 10-20 years and $28.1 \%$ had lived there less than five years. Approximately half (48.7\%) indicated not knowing any of the Finnish language, while 14 participants (11.2\%) indicated having good and 21 (16.4\%) acceptable language proficiency. The rest reported some knowledge of Finnish. About half of the Somalis (48.8\%) reported being illiterate.

\section{Use of healthcare services in Somali and Finnish groups}

The results in Table II show significant differences in the use and accessibility of healthcare services and preventive care between older Somali and Finnish individuals. The Somalis reported less use of the services of personal/family doctors in municipality healthcare centers; these physicians are also gatekeepers to specialized care in Finland. In the Finnish group, 69\% used the services of personal/family doctors, whereas the share among Somalis was $45.5 \%\left(\chi^{2}=9.03, \mathrm{p}<\right.$ 
$.001 ; \mathrm{N}=256)$. The difference between the two groups in their accessing various doctor's services was highly significant $\left(\chi^{2}=63.87, \mathrm{p}<.0001 ; \mathrm{N}=256\right)$ : Somalis used more GP services $(90 \%)$ than the Finns (34\%), whereas the Finns used substantially more private doctor services (13\%) than the Somalis (3\%). Moreover, the Somalis accessed the nurses in healthcare centers more often (97.5\%) than the Finns $(31 \%),\left(\chi^{2}=67.68, \mathrm{p}<.0001 ; \mathrm{N}=256\right)$.

Generally, the Somali migrants (9\%) used fewer preventive age-based services than the Finns (26\%). Percentages for the Somalis/Finns who reported having participated in preventive screening tests specific to females were $35 \% / 81 \%$ for mammography, $22 \% / 69 \%$ for cytology of the cervix, and $18 \% / 58 \%$ for gynecological examinations. For men, prostate palpation was reported by 7\%/27.5\% and PSA-blood sample by $10 \%$ / 28\%. Other examinations, such as blood pressure checks, were reported by 58\%/80\%, respectively. The Somalis used only vision and hearing checkups more than the Finns: $87 \%$ and $61 \%$ of the Somalis and $70 \%$ and $36 \%$ of the Finns, respectively, used these services.

The usage of mental health services was very low, $4.0 \%(\mathrm{~N}=5)$ among Somalis and $2.5 \%$ (N=3) among Finns. Thus no further analyses of the differences in accessibility were possible.

\section{Cultural preferences for mental health services}

The preferred service providers for mental healthcare among the Somali group are presented in Table III. Results indicate that $70 \%$ of the studied Somalis preferred to visit a religious expert while $21 \%$ preferred Finnish healthcare. In addition, 5.5\% preferred to turn to a traditional saar healer, while only $0.8 \%$ explicitly mentioned transnational care outside Finland. In total, $88 \%$ of the Somali participants preferred informal mental healthcare services, and $21 \%$ preferred Finnish health services.

\section{Service usage gap in Somali and Finnish elderly}


Table IV reports the associations between mental health problems and usage of healthcare services among older Somali and Finnish individuals. The results do not support the hypothesis of a service usage gap among Somali immigrants. The occurrence of high levels of mental health symptoms and non-service use was not found among the older Somali immigrants, but it was found unexpectedly among the Finnish participants concerning depressive psychological-cognitive symptoms. The significant Group X Service usage-interaction $(F(1,236)=6.62, p<01)$ indicates that the Finns who suffered from these symptoms used fewer healthcare services than non-sufferers, whereas in the Somali group, there was no significant association between depressive symptoms and health service usage. The main effects of service use reveal that, generally, the severity of mental health symptoms were not associated with service use. The group main effects revealed that the Somali migrants showed more severe psychiatric distress and somatic-affective depressive symptoms than the Finns did, but the Somali participants showed fewer psychological-cognitive depressive symptoms than the Finns.

\section{Discussion}

Our results confirmed that older Somali immigrants in Finland have less access to and usage of personal and specialized doctors' and preventive healthcare services than the native older population. The result somewhat aligns with previous research in Finland (Castaneda et al. 2012; Koponen et al. 2015) and elsewhere (Kirmayer et al. 2011) that found lower usage of specialized professionals among immigrants, especially in the psychiatric and mental health field. In our matched-pair comparison, however, both the Somali (4\%) and Finnish (2.5\%) participants reported very low access to and usage of mental health services during the previous 12 months, which prevented further analyses. We could not verify the service usage gap phenomenon, in which immigrants, despite their high needs, would use (Western) mental health services less than native persons. In general, the Somali migrants showed more psychiatric distress and somatic-affective 
symptoms of depression and used less specialized services, but a strict empirical testing of this gap in the matched-pair ethnic groups defeated the idea.

Our findings emphasize the importance of culturally salient mental health services by showing that the older Somali individuals clearly preferred culturally familiar traditional care, particularly religious healing, instead of the Finnish mental health services. The low use of mental healthcare among immigrants and refugees is commonly explained by linguistic and cultural barriers (Kirmayer et al. 2011). This is likely to apply also to the older Somali individuals in this study, whose prevailing religious and cultural views regarding healing mental distress may have resulted in the non-usage of Finnish mental healthcare. Older Somalis especially cherished religious experts, such as sheiks and imams in a mosque, for healing mental distress.

Another explanation for the low use of preventive and specialized healthcare services among immigrants relates to the health professionals' cultural insensitivity and ignorance. Immigrants' usage of Western mental health services is also hindered by the lack of knowledge on available services, which may explain the low accessibility to personal/family doctors among Somali elders. Native Finns have substantially fewer language barriers, and they are familiar with the personal doctor system. Moreover, they may be more demanding and subsequently unsatisfied with a nurse consultation.

Furthermore, those Somalis who do suffer from mental health problems are more likely to have access to, visit, and trust a GP than a psychologist or a psychiatrist. This can be explained, in part, by the fact that mental illness in Somali society has been seen as highly stigmatizing; therefore, Somalis prefer to utilize health services aimed at somatic diseases (Cavallera et al. 2016; Johnsdotter et al. 2011; Mölsä, Hjelde, and Tiilikainen 2010). This observation also concurs with a Canadian study showing that Ethiopian migrants consult GPs for their mental health problems (Fenta, Hyman, and Noh 2007). 
Importantly, there also seem to be structural barriers to healthcare. As compared to the native Finns, the Somali migrants did not have equal access to personal/family doctors. These doctors function as gatekeepers for specialized services in the Finnish healthcare system, and, therefore, the decreased access to them among Somali participants created further barriers for their healthcare service use. Instead of having direct contact with their own personal/family doctors, the older Somalis were typically first referred to consult a nurse. Therefore, the Somalis faced double barriers in trying to access special care, including mental health services.

This study does not provide explanations for the revealed structural barrier to specialized healthcare that elderly migrants experience in Finland, and it should be studied in more detail. Hence, in addition to studying the cultural barriers to the use of health services, this study suggests the need to explore also the service providers and structures and possible inequalities that may impact the use of specialized health services by migrants.

Research in Western countries suggested that established healthcare should be more sensitive to different ways of expressing and communicating distress symptoms and health problems that are typical to immigrants (Abe-Kim, Gong, and Takeuchi 2004; Tiilikainen and Koehn 2011; Wittig et al. 2008). Our findings suggest that uninformed attitudes among health professionals lead to attempts to filter 'real' patients from those who are seen as 'somatising' migrants and refugees. Public healthcare and access to a personal/family doctor would be particularly essential for older migrants, who often do not have access to private or occupational healthcare. They would benefit from a long-term care relationship with a trusted doctor in the Finnish system.

This study confirmed some previous research findings that immigrants prefer to use alternative, traditional, and culturally salient mental health services instead of Western medicalized care (Abe-Kim, Gong, and Takeuchi 2004). Concerning preventive healthcare, the study findings are in line with earlier studies indicating that migrant women tend to use available preventive 
healthcare less than the women belonging to the general population, even if the services are free of charge (Arnold, Razum, and Coeberg 2010; Morrison et al. 2012). The research also reinforces previous findings in Finland showing that, in particular, Somali women used less preventive healthcare, like cancer screenings, as compared to other female migrants (Castaneda et al. 2012). Both cultural insensitivity in the Finnish healthcare system and the cultural perceptions about prevention among Somalis contribute to this non-usage. In Somalia, health services at large have not been organized at preventing diseases but at curing them. Moreover, the use of preventive health services is regarded as meaningless, which is also succinctly captured by Somali proverbs, such as "Alla-watoo wehel uma baahna" - He who is led by Allah does not need a guide - or "Belaayo kaa sii jeedda layskuma soo jeediyo" - If a trouble shows its back, do not force it to show its face. Hence, everything is seen to be in God's hands, and, moreover, if a person does not feel sick, it is better not to visit a doctor who might detect signs of illness. Yet, it may also be that migrants tend to have lower referral rates to mammography and cervical cancer screenings in Finland, as shown through systematic review in other European countries (Norredam, Nielsen, and Krasnik 2010). It is thus easy to agree with Pavlish and colleagues that health providers of immigrant populations must understand the underlying cultural health beliefs and expectations of a population in order to generate effective healthcare interactions (Pavlish, Noor, and Brandt 2010).

Even if the Nordic welfare states like Finland provide universally-accessible healthcare, immigrants and especially those suffering from mental health problems are at risk for inadequate help. An immigrant who suffers from severe mental health problems and who does not use available services, may fall through the social safety net, including losing or failing to access their medical and social benefits. For example, an individual suffering from severe depression who lacks professional help may lose their home due to unpaid rent, further leading to a delay in treatment and diagnosis. Moreover, a Finnish resident who is disconnected from official mental health services will not be 
entitled to unemployment benefits and/or a pension. Thus, there is a need to develop an inclusive health policy for service delivery, particularly regarding mental health services. The services aimed at migrant populations should be not only available and accessible, but also acceptable and appropriate for the users. Health education designed for individuals and families of migrant backgrounds, including religious authorities such as imams, could improve migrants' health by increasing their knowledge about available services and influencing their attitudes toward those services. In particular, literacy on specific mental health symptoms, causes of mental disorders, and the effectiveness of psychiatric treatments would be valuable. Ethnic minorities should have equal access to health services, regardless of their understanding of the system and society where they live. Equal access to healthcare is a fundamental human rights issue (Koopmans et al. 2013).

We could not verify the existence of a service usage gap among Somali elders, which would have been exhibited by lower usage of health services despite higher needs, indicated by severe mental health problems. It is noteworthy that the Somali participants showed more psychiatric distress and somatic-affective depression than native Finns, who, in their part, suffered more from psychological-cognitive depressive symptoms. The cultural background thus influenced the expression of psychic suffering. Our findings also suggest it was the pattern of healthcare usage that differed between the Somali and Finnish participants, and not only the general accessibility or availability of services. Somali elders had less access to personal/family and specialized doctors and preventive healthcare, but they used more nursing services. Unexpectedly, it was rather in the Finnish group that those with high psychological-cognitive depressive symptoms used fewer general health services. The result may reflect Finnish cultural values: older Finns suffering from low self-worth, blaming themselves, and having suicidal ideas, which are typical symptoms of psychological-cognitive depression, may feel helpless and demoralized due to demands for resilience and self-sufficiency that prevailing values in Finnish society impose on them. 


\section{Limitations of the study}

The study deserves criticism for its comparative setting. First, the Somali migrants were interviewed, while the Finnish participants self-completed the questionnaires, and, moreover, the two data sets were collected at different points in time. Second, while the measures were used before in Finnish population surveys, their validity has not been thoroughly established across migrants in Finland, except for culturally-salient depressive dimensions (Kuittinen et al. 2014).

\section{Conclusions}

Compared to the Finns, the Somalis encountered substantial institutional barriers to physical and mental healthcare in spite of their greater needs. Somalis preferred the use of informal care, specifically religious healing for mental problems. In the Nordic context, there is a great need for more intervention research to understand the underlying issues that impact the use of healthcare services among the immigrants, which our study reveals as low access to preventive and specialized services and high usage of nursing services. Healthcare providers must develop holistic mental health services that are culturally understandable and acceptable to the migrant populations.

\section{Disclosure statement}

No potential conflict of interest was reported by the authors.

\section{Funding}

This study was supported by the Finnish Cultural Foundation and the Academy of Finland.

\section{Key messages}

(1) Older Somali migrants encountered institutional barriers to accessing healthcare.

(2) Older Somali migrants preferred informal mental healthcare, especially religious healing, instead of Western healthcare practices.

(3) Culturally appropriate mental health intervention efforts should focus on awareness raising regarding mental health perceptions and attitudes, and preventive healthcare. 
(4) Healthcare institutions should provide inclusive mental healthcare services that are acceptable and appropriate. 


\section{References}

Abe-Kim, Jennifer, Fang Gong, and David Takeuchi. 2004. "Religiosity, spirituality, and helpseeking among Filipino Americans: Religious clergy or mental health professionals?" Journal of Community Psychology 32 (6): 675-689.

Arnold, Melina, Oliver Razum, and Jan-Willem Coebergh. 2010. "Cancer risk diversity in nonwestern migrants to Europe: An overview of the literature." European Journal of Cancer 46 (14): 2647-2659.

Beck, A.T., C. Ward, M. Mendelson, J. Mock \& J. Erbaugh. 1961. "An inventory for measuring depression." Archives of General Psychiatry 4 (6): 561-571.

Castaneda, Anu E., Shadia Rask, Päivikki Koponen, Mulki Mölsä, and Seppo Koskinen, eds. 2012. Maahanmuuttajien terveys ja hyvinvointi-Tutkimus venäläis-, somalialais- ja kurditaustaisista Suomessa [Migrant health and wellbeing. A study on persons of Russian, Somali and Kurdish origin in Finland]. Raportti 61/2012. Helsinki: Terveyden ja hyvinvoinnin laitos.

Cavallera, V, M. Reggi, S. Abdi, Z. Jinnah, J. Kivelenge, A. M. Warsame, A. M. Yusuf, P. Ventevogel. 2016. Culture, context and mental health of Somali refugees: a primer for staff working in mental health and psychosocial support programmes. Geneva: United Nations High Commissioner for Refugees.

Close, Ciara, Anne Kouvonen, Tania Bosqui, Kishan Patel, Dermot O’Reilly, and Michael Donnelly. 2016. "The mental health and wellbeing of first generation migrants: a systematicnarrative review of reviews." Globalization and Health 12 (1): 47. doi: 10.1186/s12992-0160187-3

Derogatis, Leonard R. 1992. Symptom Checklist-90-Revised. Minneapolis, MN: NCS Pearson.

Diaz, Esperanza and Bernadette N. Kumar. (2014). "Differential utilization of primary health care services among older immigrants and Norwegians: a register-based comparative study in Norway." BMC Health Services Research 14 (1): 623. DOI: 10.1186/s12913-014-0623-0.

Fazel, Mina, Jeremy Wheeler, and John Danesh. 2005. "Prevalence of serious mental disorder in 7000 refugees resettled in western countries: A systematic review." The Lancet 365 (9467): 1309-1314.

Fenta, Haile, Ilene Hyman, and Samuel Noh. 2007. "Health service utilization by Ethiopian immigrants and refugees in Toronto." Journal of Immigrant and Minority Health 9 (4): 349-357.

Gerritsen, Annette A. M., Inge Bramsen, Walter Devillé, Loes H. M. van Willigen, Johannes E. Hovens, and Henk M. van der Ploeg. 2006. "Use of health care services by Afghan, Iranian, and Somali refugees and asylum seekers living in The Netherlands." European Journal of Public Health 16 (4): 394-399.

Gissler, Mika, Maili Malin, Petri Matveinen, Matti Savimäki, and Aki Kangasharju. 2006. Maahanmuuttajat ja julkiset palvelut: Terveydenhuollon palvelut ja sosiaalihuollon laitospalvelut. Pienten lasten hoito ja sosiaalihuollon avopalvelut [Immigrants and public services: Health care services and residential care services].Työpoliittinen tutkimus 296. Helsinki: Työministeriö.

Guerin, Bernard, Pauline Guerin, Roda Omar Diiriye, and Susan Yates. 2004. "Somali Conceptions and Expectations of Mental Health: Some Guidelines for Mental Health Professionals." New Zealand Journal of Psychology 33 (2): 59-67. 
Heistaro, Sami, ed. 2008. Methodology report: Health 2000 Survey. Helsinki: National Public Health Institute.

Johnsdotter, Sara, Karin Ingvarsdotter, Margareta Östman, and Aje Carlbom. 2011. "Koran reading and negotiation with jinn: Strategies to deal with mental ill health among Swedish Somalis." Mental Health, Religion \& Culture 14 (8): 741-755.

Joronen, Tuula and Abdirizak Hassan Mohamed. 2015. Kauppakansa pakosalla: Somaliyrittäjät meillä ja muualla [Communities of Somali refugees and entrepreneurship in different parts of the world]. Helsinki: City of Helsinki Urban Facts.

Kirmayer, Laurence J., Lavanya Narasiah, Marie Munoz, Meb Rashid, Andrew G. Ryder, Jaswant Guzder, Ghayda Hassan, Cécile Rousseau, and Kevin Pottie, for the Canadian Collaboration for Immigrant and Refugee Health (CCIRH). 2011. "Common mental health problems in immigrants and refugees: General approach in primary care." CMAJ: Canadian Medical Association Journal = Journal De l'Association Medicale Canadienne 183 (12): E959-967.

Koopmans, G. T., E. Uiters, W. Deville, and M. Foets. 2013. "The use of outpatient mental health care services of migrants vis-à-vis Dutch natives: Equal access?" The International Journal of Social Psychiatry 59 (4): 342-350.

Koponen, Päivikki, Kristiina Manderbacka, Satu Jokela, Anu Castaneda, Jaana Suvisaari, and Liisa Suominen. 2015. "Sosiaali- ja terveyspalvelujen tarve ja käyttö [Need and use of social and health care services]. In Ulkomaista syntyperää olevien työ ja hyvinvointi Suomessa 2014 [Work and wellbeing of people of foreign origin in Finland 2014], edited by Tarja Nieminen, Hanna Sutela, and Ulla Hannula, 171-184. Helsinki: Tilastokeskus.

Kuittinen, Saija, Raija-Leena Punamäki, Mulki Mölsä, Samuli I. Saarni, Marja Tiilikainen, and Marja-Liisa Honkasalo. 2014. "Depressive symptoms and their psychosocial correlates among older Somali refugees and native Finns." Journal of Cross-Cultural Psychology 45 (9): 14341452.

Ladin, Keren and Steffen Reinhold. 2013. "Mental health of aging immigrants and native-born men across 11 European countries." The Journals of Gerontology Series B: Psychological Sciences and Social Sciences 68 (2): 298-309. Doi:10.1093/geronb/gbs163.

Lewis, I. M., Ahmed Al-Safi, and Sayyid Hurreiz. 1991. Women's Medicine: The Zar-Bori Cult in Africa and beyond. Edinburgh: Edinburgh University Press, for the International African Institute.

McCrone, P., K. Bhui, T. Craig, S. Mohamud, N. Warfa, S. A. Stansfeld, G. Thornicroft, and S. Curtis. 2005. "Mental health needs, service use and costs among Somali refugees in the UK." Acta Psychiatrica Scandinavica 111 (5): 351-357.

Mirsky, Julia, Robert Kohn, Pnina Dolberg, and Itzhak Levav. 2011. "Suicidal behavior among immigrants." Social Psychiatry and Psychiatric Epidemiology 46 (11): 1133-1141.

Morrison, T. Ben, Mark L. Wieland, Stephen S. Cha, Ahmed S. Rahman, and Rajeev Chaudhry. 2012. "Disparities in preventive health services among Somali immigrants and refugees." Journal of Immigrant and Minority Health 14 (6): 968-974.

Mölsä, Mulki Elmi, Karin Harsløf Hjelde, and Marja Tiilikainen. 2010. "Changing conceptions of mental distress among Somalis in Finland." Transcultural Psychiatry 47 (2): 276-300.

Mölsä, Mulki, Raija-Leena Punamäki, Samuel I. Saarni, Marja Tiilikainen, Saija Kuittinen, and Marja-Liisa Honkasalo. 2014. "Mental and somatic health and pre- and post-migration factors among older Somali refugees in Finland." Transcultural Psychiatry 51 (4): 499-525.

Norredam, Marie, Signe S. Nielsen, and Allan Krasnik. 2010. "Migrants' utilization of somatic healthcare services in Europe-a systematic review." European Journal of Public Health 20 (5): 555-563. Doi: 10.1093/eurpub/ckp195. 
Omar, Mayesh A. 1986. "Developments in mental health services in Somalia." NIMHANS Journal 4 (2): 131-132.

Pavlish, Carol Lynn, Sahra Noor, and Joan Brandt. 2010. "Somali immigrant women and the American health care system: Discordant beliefs, divergent expectations, and silent worries." Social Science \& Medicine 71 (2): 353-361.

Sandvik, Hogne, Steinar Hunskaar, and Esperanza Diaz. 2012. "Immigrants' use of emergency primary health care in Norway: A registry-based observational study." BMC Health Services Research 12 (308). Available at http://bmchealthservres.biomedcentral.com/articles/10.1186/1472-6963-12-308.

Serkkola, Ari. 1994. A Sick Man is Advised by a Hundred. Pluralistic Control of Tuberculosis in Southern Somalia. Kuopio University Publications D. Medical Sciences 40. Kuopio: University of Kuopio, Department of Public Health.

Somalis in Helsinki. 2013. Somalis in European Cities. New York and London: Open Society Foundations.

Statistics Finland. 2016. Population structure: Language according to age and sex by region 1990-2015. Available at http://pxnet2.stat.fi/PXWeb/pxweb/en/StatFin/StatFin__vrm_vaerak/030_vaerak_tau_102.px /?rxid=5392dbb9-7dfc-4802-9d67-aa72388f3686 (retrieved January 26, 2017).

Tiilikainen, Marja and Peter H. Koehn. 2011. "Transforming the Boundaries of Health Care: Insights from Somali Migrants." Medical Anthropology 30 (5): 518-544.

Uiters, Ellen, Walter Devillé, Marleen Foets, Peter Spreeuwenberg and Peter P. Groenewegen. 2009. "Differences between immigrant and non-immigrant groups in the use of primary medical care; a systematic review." BMC Health Services Research 9 (1): 76. Doi:10.1186/1472-6963-9-76.

Vervliet, Marianne, Melinda A. Meyer Demott, Marianne Jakobsen, Eric Broekaert, Trond Heir, and Ilse Derluyn. 2014. "The mental health of unaccompanied refugee minors on arrival in the host country." Scandinavian Journal of Psychology 55 (1): 33-37. Doi: 10.1111/sjop.12094.

Wahlbeck, Kristian, Kristiina Manderbacka, Lauri Vuorenkoski, Hannamaria Kuusio, Minna-Liisa Luoma, and Eeva Widström. 2008. Quality in and Equality of Access to Healthcare Services: Country Report for Finland. Helsinki: Stakes.

Warfa, Nasir, Kamaldeep Bhui, Tom Craig, Sarah Curtis, Salaad Mohamud, Stephen Stansfeld, Paul McCrone, and Graham Thornicroft. 2006. "Post-migration geographical mobility, mental health and health service utilisation among Somali refugees in the UK: A qualitative study." Health \& Place 12 (4): 503-515.

Wedel, Johan. 2011. "Mental Health Problems and Healing among Somalis in Sweden." Bildhaan 11: 73-89.

Whitley, Rob, Laurence J. Kirmayer, and Danielle Groleau. 2006. "Understanding immigrants' reluctance to use mental health services: a qualitative study from Montreal." The Canadian Journal of Psychiatry 51 (4): 205-209.

Wittig, U., J. Lindert, M. Merbach, and E. Brähler. 2008. "Mental health of patients from different cultures in Germany." European Psychiatry 23: 28-35. 
Table I. Background variables in Somali and Finnish groups

\begin{tabular}{|c|c|c|c|c|}
\hline & \multicolumn{2}{|c|}{$\begin{array}{l}\text { Somalis } \\
(n=128)\end{array}$} & \multicolumn{2}{|c|}{$\begin{array}{c}\text { Finns } \\
(n=128)\end{array}$} \\
\hline & $\%$ & $\mathrm{~N}$ & $\%$ & $\mathrm{~N}$ \\
\hline \multicolumn{5}{|l|}{ Gender } \\
\hline Women & 59 & 75 & 58.6 & 75 \\
\hline Men & 41.4 & 53 & 41.4 & 53 \\
\hline \multicolumn{5}{|l|}{ Age } \\
\hline $50-59$ years & 60.9 & 78 & 60.9 & 78 \\
\hline $60-69$ & 33.6 & 43 & 34.4 & 44 \\
\hline $70-80$ & 5.5 & 7 & 4.7 & 6 \\
\hline \multicolumn{5}{|l|}{ Civic status } \\
\hline Married & 62.2 & 79 & 61.7 & 79 \\
\hline Divorced & 9.4 & 12 & 9.4 & 12 \\
\hline Widow & 26 & 33 & 26.6 & 34 \\
\hline Single & 2.4 & 3 & 2.3 & 3 \\
\hline \multicolumn{5}{|l|}{ Education } \\
\hline No education & 65.6 & 84 & 62.5 & 80 \\
\hline Various courses & 8.6 & 11 & 13.3 & 17 \\
\hline Vocational school & 9.4 & 12 & 7.8 & 10 \\
\hline Polytechnic school & 7.8 & 10 & 7.8 & 10 \\
\hline University & 5.5 & 7 & 5.5 & 7 \\
\hline Other & 3.1 & 4 & 3.1 & 4 \\
\hline \multicolumn{5}{|l|}{ Employment } \\
\hline Full-time employment & 28.2 & 36 & 78.1 & 100 \\
\hline Part-time employment & 12.4 & 16 & 9.4 & 12 \\
\hline Unemployed & 59.4 & 76 & 12.5 & 16 \\
\hline
\end{tabular}

Note: Somalis and Finns are pair-matched groups according to age, gender, civic status and education. 
Table II. The usage of health care services in the Somali and Finnish groups (\% of yes answers)

Somalis Finns

$\% \quad \mathrm{~N} \quad \% \quad \chi^{2 \text {-value }}$

Somatic health

Usage of personal/family doctor for help

$45.5 \quad 51$

68.7

46

$9.03^{* *}$

Accessibility to doctor's services

General practitioner, GP

$89.8 \quad 79$

34.0

$63.87 * * * *$

Occupational health care

$\begin{array}{llll}0 & 0 & 47.2 & 25\end{array}$

Private doctor

$3.4 \quad 3$

13.2

7

Outpatient clinic in hospital

4

3.8

2

Own doctor (friend, relative)

2

1.9

1

Accessibility to nurse's services

Health care center

$97.5 \quad 78$

31.3

15

Occupational health care

$0 \quad 0$

54.2

26

Other

2.5

2

14.6

7

Preventive health care servives

$\begin{array}{lccccc}\text { Age-group check-up } & 9.2 & 7 & 26.0 & 32 & 8.42 * * \\ \text { Vision check-up } & 86.8 & 79 & 69.6 & 87 & 8.77 * * \\ \text { Hearing check-up } & 60.7 & 54 & 36.0 & 45 & 12.73 * * * * \\ \text { Blood pressure } & 57.8 & 52 & 80.0 & 100 & 12.47 * * * \\ \text { Blood sugar } & 57.3 & 51 & 56.1 & 69 & 0.03 \\ \text { Cholesterol measure } & 46.1 & 41 & 65.6 & 82 & 8.11^{* *} \\ \text { Bone density } & 16.5 & 14 & 5.6 & 7 & 6.54 * \\ \text { Mammography (women) } & 35.4 & 23 & 81.1 & 60 & 30.04 * * * * \\ \text { Breast palpation (women) } & 25.0 & 15 & 38.9 & 28 & 2.87\end{array}$




\begin{tabular}{lrrrrr}
\hline Breast ultrasonic examination & 14.8 & 9 & 5.6 & 4 & 3.16 \\
Pap test (cytology of the cervix) & 22.2 & 14 & 69.4 & 50 & $30.05^{* * * *}$ \\
Gynegological examination & 18.3 & 11 & 58.3 & 42 & $21.79 * * *$ \\
Prostate palpation (men) & 7.4 & 5 & 27.5 & 14 & $8.77^{* *}$ \\
Prostate ultrasonic examination (men) & 8.7 & 6 & 13.7 & 7 & 0.77 \\
PSA- blood sample (prostate indicator) & 10.3 & 7 & 28.0 & 14 & $6.17 *$ \\
\hline
\end{tabular}

Note: $* \mathrm{p}<.05 ; * * \mathrm{p}<.01 ; * * * * \mathrm{p}<.0001$

Table III. The preferable service places for mental health care in the Somali group

\begin{tabular}{lcc}
\hline Source of care & $\%$ & $\mathrm{~N}$ \\
\hline Religious expert & 70.3 & 90 \\
Traditional healer (saar) & 5.5 & 7 \\
Herbalist & 12.5 & 16 \\
Finnish health care & 21.1 & 27 \\
Elsewhere & 0.8 & 1 \\
& & \\
\hline
\end{tabular}

Note: The participants were given the possibility to provide two preferences, which explains that the total does not account to $100 \%$. 